\title{
Manejo sostenible y sustentable de fincas productoras mediante procesos participativos en Sáchica, Boyacá
}

\section{Land plantations sustainable managing by participative processes in Sáchica, Boyacá}

Fecha de Recepción: 16 de noviembre de 2012 Fecha de Aceptación: 24 de octubre de 2013
Ángel Eduardo Ramírez Amaya', Germán Gonzalo Hurtado²

\section{Resumen}

Objetivo. Elaborar un proyecto de desarrollo sostenible y sustentable de fincas productoras mediante procesos participativos en el municipio de Sáchica, Boyacá. Materiales y métodos. La investigación se realizó con familias campesinas de la vereda Arrayán Alto, del municipio de Sáchica, Boyacá, mediante la metodología Investigación Acción Participativa (IAP), que se centra en la participación de las comunidades para elaborar propuestas concertadas con ellas. El trabajo se desarrolló en varias fases, que incluyeron un diagnóstico socioeconómico de las familias, capacitaciones y concientización en temas relacionados con la agricultura ecológica y de granjas integrales. Resultados. Se elaboró un plan de trabajo que permitió la construcción

\begin{abstract}
Objective. To elaborate a sustainable productive project development, among the rural families of the small village Alto Arrayán, located in the Sáchica Boyacá municipality. Materials and methods. The methodology is denominated Investigation, Action, Participating (IAP), which enables the community to elaborate proposals concerted among them by the joint participation's principles. An investigation was carried out in several phases, which included the family's socioeconomic diagnosis, enabling them to become conscious, in several topics related with the integral farms's ecological agriculture. Results. After several proposals, a final document was made, which has been sent to some institutions such as the Municipal Governorship, in order to
\end{abstract}

1 Ingeniero Agrónomo. Profesional Independiente. eduardoorganic20@hotmail.com

2 Ingeniero Agrónomo, Ph.D. Corporación CEI3TAS. docente.fundación-cidboy@yahoo.com. 
de un documento final que ha servido para el apoyo logístico o económico de las entidades gubernamentales locales para la instalación y plantación técnica del cultivo de gulupa con familias de la vereda Arrayán Alto.

Palabras clave: Desarrollo sostenible y sustentable, granjas integrales, agricultura ecológica (Fuente: USDA). obtain logistical or economical support, to build the technical plantation premises, for the Gulupa cultivation.

Key words: Sustainable development, integral farms, ecological agriculture (Source: USDA). 


\section{Introducción}

Actualmente, en el sector agropecuario predomina la agricultura itinerante, realizada durante años bajo sistemas rudimentarios y antieconómicos, de baja productividad y alto costo ecológico, cuyos efectos más nocivos son la erosión y la pérdida de la capacidad nutricional del suelo (1). El nuevo orden mundial exige nuevas alternativas de producción, bajo un enfoque menos negativo con nuestro medioambiente a largo plazo; ello implica una nueva concepción del mundo y de la vida, que posibilita hallar alternativas socialmente viables con el rigor científico y técnico (2). Existen técnicas de agricultura que causan un mínimo impacto al medio y trabajan de la mano con las comunidades mediante investigaciones participativas (3); dentro de estas se encuentra la agricultura ecológica, que agrupa diversas técnicas, tales como la labranza mínima, los abonos orgánicos, las coberturas verdes y los controles biológicos para plagas y enfermedades, entre otras, que conducen a un desarrollo más justo con el ambiente (4).

De lo anterior surge la iniciativa de desarrollar una propuesta de manejo sostenible y sustentable de fincas productoras mediante procesos participativos en Sáchica, Boyacá, municipio caracterizado por la explotación agrícola intensiva, donde el 92\% de sus pobladores viven del cultivo de la cebolla de bulbo. Se pretende realizar conjuntamente un diagnóstico detallado de la situación económica y social del productor bajo los principios de la IAP -Investigación Acción Participativa- (5).

\section{Métodos y participantes}

El proyecto se desarrolló por medio de la Investigación Acción Participativa (IAP), que integra a las comunidades en la toma de decisiones y trabaja con un esquema de recolección de información cualitativa y cuantitativa (5), y comprendió varias fases, que incluyeron un diagnóstico socioeconómico de las familias, capacitaciones y concientización en temas relacionados con la agricultura ecológica y de granjas integrales.

Fase I, Diagnóstico socioeconómico. La situación social y económica de las 20 familias campesinas de la vereda Arrayán Alto se conoció por medio de entrevistas personales.

Fase II. Trabajo de Campo. Una vez realizado el diseño, tuvo lugar el trabajo de campo; se trató de una forma de proceder que, a través de la observación sobre el terreno y la profundización en situaciones y campos particulares, ofreció la posibilidad de obtener un conocimiento de fenómenos, hechos y problemas inaccesibles con solo la revisión bibliográfica.

Fase III. Reunión entre instituciones y familias implicadas en el proyecto, para conocer puntos de vista, intereses que encierra la investigación y ajustar sus detalles; asimismo, se dejó en claro la necesidad de la participación de todos los implicados en esta investigación. Para recolectar la información se determinaron aspectos de tipo económico (mano de obra, fuentes de ingresos, recursos, actividades económicas) y social (conformación familiar, alimentación diaria, formas de organización social, educación) que presentan estos productores en sus fincas. Para acceder a esta información y alcanzar una comprensión del objeto de estudio se utilizaron diversos procedimientos y técnicas de recolección de datos, tales como entrevistas, conversaciones autoexploratorias, registro anecdótico, análisis de documentos, encuestas, observación de situaciones, reuniones de grupo, visitas a familias y mapa del estado inicial de las fincas, entre otras; al final de este proceso se analizaron los resultados de forma cuantitativa. La utilización de estas técnicas y estrategias permite obtener un conocimiento exhaustivo de todas las variables que influyen en la realidad de las familias, y concretamente en aquellos problemas a que refiere la elaboración de esta propuesta.

Fase IV. Discusión y elaboración de la propuesta (Plan de Acción). Una vez considerados los resultados de este diagnóstico y teniendo en cuenta las fortalezas y debilidades detectadas, con la ayuda de la UMATA municipal y algunos líderes comunitarios de la vereda, se convocó a una serie de reuniones en donde toda la comunidad discutió, debatió y participó para la elaboración de un plan de acción. La comunidad planteó acciones por seguir y fue autogestora de su desarrollo. Se realizaron capacitaciones y se dio la oportunidad 
de encuentro con la comunidad para trabajar en el objetivo del proyecto; en cada taller se trabajó en la planificación de acciones por seguir.

\section{Resultados y discusión}

Diagnóstico socioeconómico de las familias campesinas: El $40 \%$ de las familias campesinas con las que se desarrolló el proyecto no cuentan con un área de terreno productivo mayor a una hectárea; solo el $5 \%$ posee un área mayor a 3 hectáreas. Para su alimento básico, las familias siembran cereales (maíz, trigo, cebada), legumbres (arveja, haba, fríjol), hortalizas (cebolla, tallos, zanahoria, repollo, cilantro, ahuyama y calabaza, entre otras) y frutales, principalmente caducifolios (durazno, freijoa y manzana) y tomate de árbol. En la mayoría de los casos, las familias destinan sus suelos a sembrar cebolla cabezona, cuya venta les proporciona el dinero para comprar en el mercado local productos como harinas, granos, café, sal, frutas y carnes, necesarios para su dieta familiar.

El consumo diario de carne, del grupo familiar, es menor a una libra, en el $60 \%$ de los casos; igualmente el de verduras, en el $55 \%$ de las familias entrevistadas; el $70 \%$ de los hogares campesinos consumen diariamente, en promedio, una botella de leche, para el grupo familiar; un $20 \%$ no consume y solo el $10 \%$ consume entre 1 y 2 botellas. El consumo de alimentos por el grupo familiar tiene especial relevancia, si se toma en cuenta el número de personas que lo conforman; se observa que solo el $30 \%$ de los hogares encuestados tienen entre 1 y 3 integrantes, y que el $70 \%$ cuentan con más de 4 individuos por familia. En cuanto a la edad promedio de la población, se encontró que el $48.8 \%$ del total corresponde a edades no superiores a los 18 años, el $44.8 \%$ se encuentra entre los 19 y 55 años, y solo el $6.4 \%$ es mayor de los 56 años de edad.

Teniendo en cuenta los resultados en la disponibilidad de alimentos y el número de integrantes del grupo familiar, se observa que se tiene un mayor acceso a los carbohidratos y, en menor proporción, a las proteínas y vitaminas, debido principalmente al factor económico que limita la compra de carne (res, pollo, pescado, etc.) y de frutas, pues estos alimentos son más costosos que los cereales y tubérculos, de uso diario más prevalente. Estos resultados son similares a los obtenidos en la encuesta nacional de demografía y salud (ENDS) 2005, en donde se encontró que en el sector rural más del $60 \%$ de las personas tienen un consumo muy bajo de frutas y verduras, mientras que para productos como la carne y la leche es de solo el $25 \%$ (6).

Propuesta de mejoramiento. El sector agrícola nacional se ha caracterizado por sus recursos naturales y sus fuentes hidrográficas, que han venido siendo disminuidas por falta de conocimiento, compromiso y manejo tanto del sector público como del privado. Las prácticas inadecuadas de explotación agrícola han dejando a un lado el desarrollo sostenible y sustentable de toda la región. El atraso económico y la falta de inversión social en el campo ha empeorado esta situación y mermado los recursos naturales; la falta de políticas y proyectos gubernamentales que generen desarrollo y progreso para el campo ha aumentado la pobreza y la migración del campesino a las grandes ciudades (7).

Se hace de vital importancia la planificación de la finca, incluyendo un mapa base con los suelos de la finca y una planificación definida que contenga todas las actividades que en ellos se desarrollan dentro de un correcto uso de la tierra y con énfasis en el control de la erosión e irrigación. Esta planificación de la finca deberá ser elaborada por el asistente técnico y el agricultor, conjuntamente; solamente así se podrá acabar con la rutina de sembrar en terrenos impropios y un solo cultivo por lustros y décadas, al final de lo cual el resultado es el desgaste de las tierras y el agotamiento paulatino del agricultor (8). 


\section{Referencias bibliográficas}

(1) Shejtman A. Economía campesina: especificidad, articulación y diferenciación. Economía campesina y agricultura empresarial; 2010.

(2) Altieri M. Bases científicas de la agricultura alternativa. Universidad de California, Berkeley, Estados Unidos; 1983.

(3) Instituto Colombiano Agropecuario ICA. Normatividad fitosanitaria para exportación de frutas frescas. División Sanidad Vegetal; 2000.

(4) Restrepo J. La teoría de la trofobiosis. Plantas enfermas para el uso de venenos en la agricultura, Cali; 2000.

(4) Fals-Borda O. Historia de la cuestión agraria en Colombia. Carlos Valencia Editores, Bogotá; 1982.
(5) Profamilia. La situación del progreso social Colombiano a comienzos del siglo XXI. Tendencias sociales a largo plazo (en línea) 2005 (fecha de acceso 10 de mayo de 2012). URL disponible en www.profamilia.org.co/ encuestas/00resumen/01general.htm

(6) Ardila J. Temas y oportunidades para la investigación agropecuaria en América Latina y el Caribe. PROCISUR-IICA; 2009.

(7) Cepeda E. Futuro para el campo. Cartilla para UMATAS. Tomo I Ciencia y tecnología para el campo. Graphos, Santa Fe de Bogotá; 1996. 